\title{
supervivencia de Rhizophora mangle L. en el manglar de Barra de Tecoanapa, Guerrero, México
}

\author{
Cristian Tovilla Hernández ${ }^{1}$ \\ D. Edith Orihuela Belmonte ${ }^{1}$
}

\begin{abstract}
RESUMEN
En el manglar de Barra de Tecoanapa, Guerrero, se estudió la fenología reproductiva, establecimiento de los propágulos y supervivencia de las plantas hasta alcanzar la primera floración en Rhizophora mangle. Se observó la producción continua de flores y propágulos a lo largo del año, con estacionalidad marcada en la época seca (máximo) y de lluvias (mínimo). La producción anual en los bosques fue de 101.7 flores $/ \mathrm{m}^{2}$ y 2.1 propágulos $/ \mathrm{m}^{2}$. La duración completa de la floración hasta la maduración de los

$D E$ 33) días; de los que 226 (DE 29) días comprenden el desarrollo de los propágulos. De 2043 flores marcadas se obtuvieron 394 propágulos, siendo la etapa de floración y la época seca las más críticas, durante las que se perdió hasta un $45 \%$ de flores iniciales (prueba Spearman). El establecimiento de los propágulos se realizó en $17(D E 5)$ días con un $96 \%$ de éxito, a los 58 y 73 meses alcanzaron la floración, con el 16 y $28.5 \%$ de las plantas en sombra y sol respectivamente (Log-rank test), observando el mejor desarrollo, en términos de altura alcanzada, en las expuestas al sol. La influencia de las mareas fue un factor decisivo, mientras que la aparición de las ramas y raíces aéreas determinó la supervivencia en los sitios.
\end{abstract}

PALABRAS CLAVE:

Estacionalidad, fenología, germinación, manglar, propágulos, supervivencia.

\begin{abstract}
The reproductive phenology, germination, mortality and survivorship of plants of Rhizophora mangle L. up to their first flowering were studied. A continuous production of flowers and propagules along the year was observed, with a marked seasonality between the dry and rainy seasons. The annual production of flowers and propagules was of $101.7 / \mathrm{m}^{2}$ and $2.1 / \mathrm{m}^{2}$, respectively. The interval from flowering through maturation of propagules was 357 (DS 33) days, of which 226 (DS 29) days comprised propagule development. Out of 2043 marked flowers, 394 propagules were obtained, the flowering stage and the dry season being the most critical, during which up to $45 \%$ of the flowers initially formed were lost (Spearman test). Germination took place at 17(DS 5) days after establishment, with a $96 \%$ success, reaching eventually the flowering stage $16 \%$ and $28.5 \%$ of the plants planted in shade and full sun, respectively (Log-rank test) The first flowering was recorded at 58 and 73 months respectively. The best development was reached in plants exposed to full sun. The influence of tides was a decisive factor, while the appearance of branches and aerial roots determined the survivorship of the plants in each site.
\end{abstract}

KEY WORDS:

Seasonality, phenology, germination, mangrove, propagules, survivorship. 


\section{INTRODUCCIÓN}

Rhizophora mangle L. tiene una amplia distribución en las costas del Océano Pacífico y el Atlántico. Esta distribución es el resultado del proceso de colonización del género Rhizophora hacia el continente americano durante los últimos 60 millones de años. A través de este tiempo, la especie ha desplegado una serie de estrategias para colonizar nuevas áreas, entre las cuales están: producción permanente de flores y propágulos (Domínguez et al., 1998), capacidad de dispersión por la corriente y mareas, facilidad de arraigamiento de los propágulos en cualquier sustrato y resistencia de estas estructuras a estar a la deriva durante mucho tiempo (Chapman, 1974; Mepham, 1983).

Se han llegado a observar propágulos viables después de un año de haberse desprendido de los árboles, y propágulos viables flotando a cientos de kilómetros mar adentro (Gill y Tomlinson, 1971; Rabinowitz, 1978; Tovilla, 1998). Los propágulos presentan una gran reserva de tejido nutritivo y un estado avanzado de desarrollo del embrión por lo que, al arraigarse en el sustrato, rápidamente puede aparecer el primer par de hojas y la planta puede depender de su reserva de nutrientes durante el primer año exclusivamente.

Aún cuando la producción de flores y propágulos es permanente, se observan fuertes variaciones a lo largo del año (Tovilla, 1998). Este mismo patrón se observa en el establecimiento de los propágulos en los bosques; mientras que la supervivencia depende de otros factores como la cantidad de luz, nivel de inundación, mareas y cantidad de nutrientes en cada sitio (Clarke y Allaway, 1993; Clarke, 1995; McKee, 1995a, b). Pocas investigaciones han analizado la floración, ropágulos y estacionalidad en las especies de mangles de floración permanente (Gill y Tomlinson, 1971;
Rabinowitz, 1978; Christensen, 1978; Jiménez, 1988) y menos aún para conocer la historia de vida de las plántulas hasta la primera floración en el campo (Tovilla, 1998).

\section{ÁREA DE ESTUDIO}

Este trabajo se realizó en dos sitios del humedal de Barra de Tecoanapa, ubicado en la desembocadura del Río Quetzala (98 $\left.45^{\prime} 04^{\prime \prime} \mathrm{W}, 16^{\circ} 30^{\prime} 12^{\prime \prime} \mathrm{N}\right)$ (Fig. 1). El clima es de tipo Aw3" (f), cálido semiseco, con dos estaciones al año: época de lluvias de junio a octubre y temporada seca de noviembre a mayo. La máxima precipitación se registra en septiembre, con registros anuales menores a $1000 \mathrm{~mm} / \mathrm{año}$. Este sistema cubre una extensión de 2062 ha, 915 ha ocupadas por un manglar, constituido por: Rhizophora mangle, Avicennia germinans, Laguncularia racemosa y Conocarpus erectus.

En los 19 años anteriores a 1990, en la zona de Barra de Tecoanapa, las precipitaciones promediaron $600 \mathrm{~mm} / \mathrm{año}$ (SARH, 1987), con un patrón de cuatro años muy secos por dos moderadamente lluviosos. Este patrón cambió en forma sustancial de 1990 a 1994; periodo en que la lluvia alcanzó hasta $1110 \mathrm{~mm} / \mathrm{año}$, con las precipitaciones más copiosas en 1991 y 1992 (1 212 mm/año). Durante el estudio se presentaron lluvias esporádicas en diciembre, enero y febrero, como sucedió en 1991, 1993 y 1994. La temperatura promedio en el área de estudio fue de $28.9{ }^{\circ} \mathrm{C}$, con una mínima de $19.4^{\circ} \mathrm{C}$, en enero y febrero; mientras que en mayo se registraron las máximas con $37.8^{\circ} \mathrm{C}$.

El área presenta mareas mixtas semidiurnas, con una altura promedio, en julio y agosto, de $0.63 \mathrm{~m}$, mientras que en marzo-abril ésta se eleva hasta $0.96 \mathrm{~m}$. Durante la pleamar se introduce una gran cantidad de agua marina en todo el manglar. Los promedios de los niveles de 

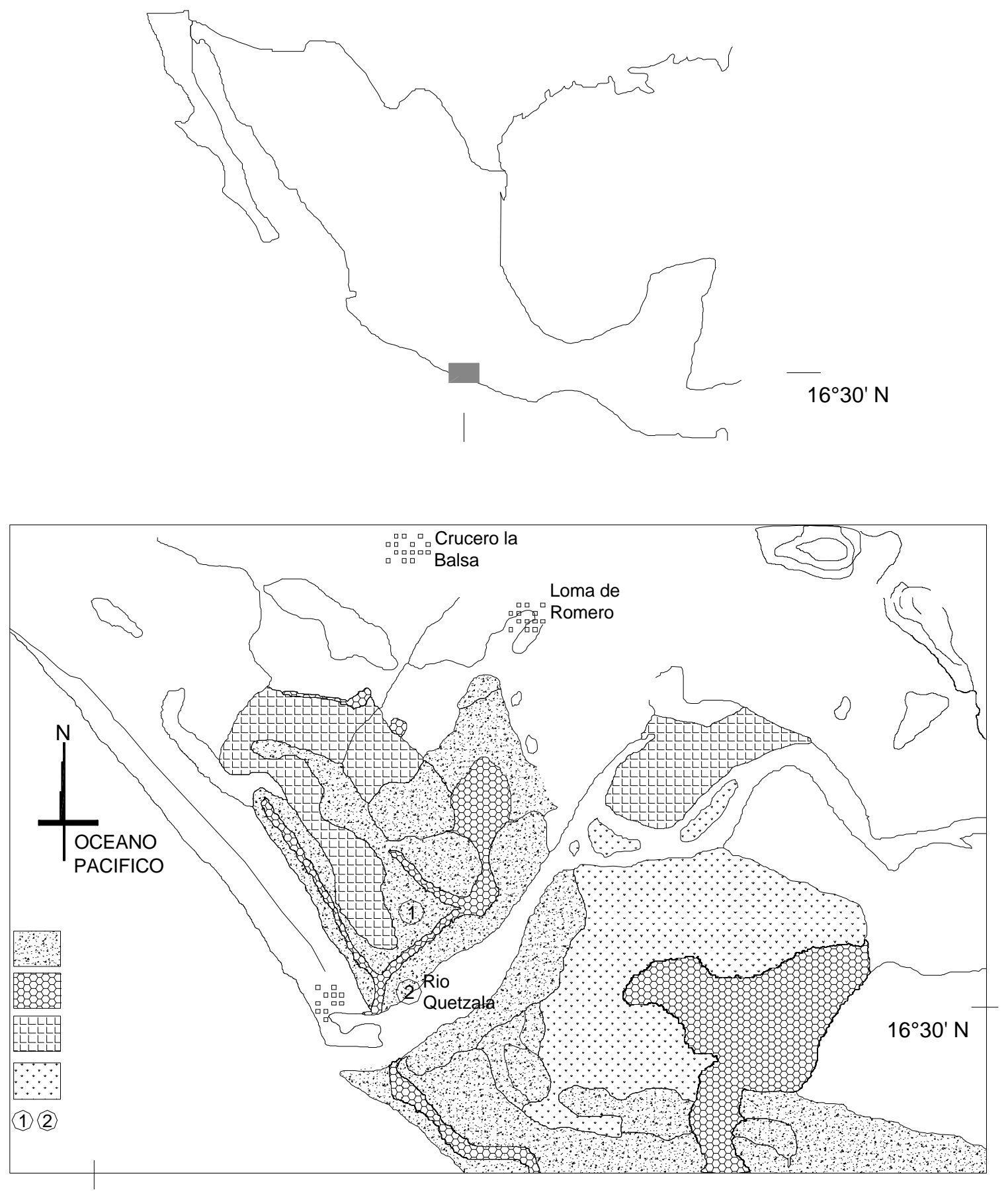

Figura 1. Ubicación del área de estudio. 
por el contrario, en septiembre esta situación se invirtió (más de $50 \mathrm{~cm}$ y 0 ).

\section{OBJETIVO}

Conocer la fenología reproductiva de $R$. mangle, la velocidad y eficiencia de la producción de flores y frutos, el establecimiento de los propágulos y la supervivencia de las plantas hasta alcanzar la primera floración, bajo dos condiciones de iluminación y una de inundación en el campo.

\section{METODOLOGÍA}

\section{Estacionalidad en la producción de flores y propágulos en el campo}

Para conocer la estacionalidad y cantidad de flores y propágulos producidos en un metro cuadrado, se utilizaron canastas colectoras. Las mediciones se iniciaron en junio de 1990 y concluyeron en agosto de 1995 en dos bosques ribereños, con características similares en cuanto a densidad y desarrollo de los árboles adultos. En cada sitio se colocaron 30 canastas colectoras de malla nylon con entramado de $1 \mathrm{~mm} x$ $1 \mathrm{~mm}$, construidas sobre marcos de madera de $0.5 \mathrm{~m} \mathrm{X} 0.5 \mathrm{~m}$, fijas a $2.5 \mathrm{~m}$ de altura y distribuidas al azar en una superficie de $2500 \mathrm{~m}^{2}$ (Cintrón y Schaeffer, 1981). El material caído se colectó mensualmente, contabilizando el número de flores y propágulos por colector, expresado por unidad de área $\left(\mathrm{m}^{2}\right)$. En cada sitio se estimó el área de la boles y la densidad/ hectárea. Debido a que las copas no son simétricas, a cada uno de los 25 árboles, se le midió el diámetro mínimo y máximo de la copa, y ambos se promediaron para obtener el área a partir de un círculo (Cintrón y Schaeffer, 1981; Tovilla, 1998).

\section{Eficiencia de la floración y tiempo de producción de propágulos}

Cada dos meses, de marzo de 1993 a enero de 1994, se marcó un lote de 100 inflorescencias con dos repeticiones hasta completar 600, distribuidas en 20 árboles a lo largo de un canal. En cada inflorescencia se colocó una etiqueta de plástico $(4 \quad \mathrm{~cm} \quad x \quad 10 \quad \mathrm{~cm})$ numerada progresivamente y de un color diferente para distinguirlas de las etiquetas de los meses anteriores. En cada etiqueta se anotó el número inicial de flores cerradas por cada inflorescencia. Cada diez días se revisaron las inflorescencias etiquetadas de cada color, anotando el número de flores abiertas y cerradas que aún permanecían en los tallos, así como los frutos y propágulos desarrollados. El experimento culminó cuando

propágulos maduros marcados se desprendieron de los árboles. Se aplicó un análisis de Spearman para conocer la correlación entre flores cerradas/flores polinizadas/frutos formados/propágulos

\section{Establecimiento de propágulos y sobrevivencia de plantas en campo}

Se colectaron 800 propágulos recién $R$. mangle, de los cuales se obtuvo el peso y longitud. En enero de 1991 se sembraron los propágulos en lotes de un área de $40 \mathrm{~m}^{2}$ cada uno, bajo dos condiciones diferentes de iluminación; en cada lote se sembraron 200 propágulos con una réplica, para un total de cuatro lotes, con una distancia entre los lotes de $5 \mathrm{~m}$, con un espaciamiento inicial entre los propágulos de $0.25 \mathrm{~m}$ y una densidad 5 propágulos $/ \mathrm{m}^{2}$. El primer tratamiento con su repetición se ubicó bajo un bosque 
inundado con una cobertura aproximada del $50 \%$ (DE 2.3) de luz, medida con un fotómetro LCD RS232. El segundo tratamiento se ubicó a $150 \mathrm{~m}$ del sitio anterior en un área totalmente expuesta $\mathrm{e}$ inundada (10 cm DE 5), en promedio, durante siete meses por año. A partir de la emergencia del primer par de hojas, bimestralmente se midieron la altura y el número de plantas sobrevivientes. Esta experiencia se repitió en ambos sitios hasta que el $50 \%$ de las plantas alcanzó la primera floración, comparando la sobrevivencia para ambos lotes a través de una prueba de Log-rank.

\section{RESULTADOS}

\section{Estacionalidad en la producción de flores y propágulos}

Entre 1990 y 1995, se observó una disminución del $12.3 \%$ durante los meses más secos (febrero-abril), en la cantidad de flores colectadas, con respecto al periodo de lluvias. Esta disminución fue más acentuada en abril de 1992 y 1993, donde se estimó una disminución del 28.3\% (Tabla 1). Durante las Iluvias (junio, julio, agosto y septiembre) se registró un aumento en la floración, proceso que se prolongó hasta enero. El promedio anual general de flores colectadas fue de 96.3 flores $/ \mathrm{m}^{2}$ (DE 35.9) siendo los años más fecundos los de 1991 y 1994. Con excepción de 1990-91, se contabilizó la mayor producción de flores en la época de lluvias.

La producción de propágulos presentó un promedio de 2.3 por metro cuadrado, una varianza de 13.9, DE 3.7, entre lluvias (junio, julio, agosto y septiem-bre) y secas (octubre, noviembre, diciem-bre, enero, febrero, marzo, abril y mayo). Durante la época seca la producción de estas estructuras disminuyó en forma sustancial (53.4\%). Por el contrario, con las lluvias se registró una gran cantidad de propágulos en los árboles, el agua y el suelo; este proceso fue así en todos los años, con excepción de 1994 donde la producción fue continua a lo largo de todo el año (Tabla 1). El año 1992 fue $\mathrm{el}_{2}$ más productivo con 4.8

A un proceso de floración masiva en el periodo julio-octubre, correspondió otro de producción elevada de propágulos, 10 a 12 meses más tarde, como sucedió en los periodos 1991-92 y 1993-94. La cobertura foliar promedio de los 50 árboles medidos en ambos sitios fue de $9.8 \mathrm{~m}^{2}(D E 6.9)$ y la densidad fue de 1 220 árboles/ha; existe un solapamiento en las coberturas foliares (no estimado) en los bosques estudiados. Con estos datos se estimó la producción de flores y propágulos aportada en el bosque (Tabla 2). La mayor producción de propágulos se registró siempre a la orilla de los canales, mientras que al interior del bosque solo se registraron propágulos de tamaño pequeño y cantidades hasta un $25 \%$ menores al total estimado por árbol.

\section{Eficiencia de la floración y tiempo de producción de propágulos}

La floración en Barra de Tecoanapa presentó una duración promedio de 135 días ( $D E 27)$, desde la formación de los primordios florales hasta el inicio de la formación del fruto. Las flores inicialmente se encuentran cerradas y en posición invertida; posteriormente, durante los siguientes 56 días ( $D E$ 11), se abren (Tabla 3). Las flores están constituidas por cuatro pétalos coriáceos (perianto) de color blanco-amarillento, que se endurecen a medida que las flores maduran. Cuando las flores se abren no poseen olor, siendo el viento el factor que facilita la polinización. Sólo se registraron hormigas sobre las flores en algunos meses.

Después de la polinización, durante los siguientes 79 días ( $D E$ 16), se constituyó el fruto y a partir de éste los propágulos; en la Tabla 3 se observa el número de días para cada evento, en los 
meses donde se marcaron lotes de flores. Se registraron diferencias en la duración de la floración, siendo en julio de 1993 cuando se determinó el tiempo máximo de 143 días ( $D E 12)$ y, en septiembre, el que resultó tiempo mínimo con 123 días (DE 9$)$.

Tabla 1. Caída de flores y propágulos $/ \mathrm{m}^{2}$ colectados en $R$. mangle, en dos bosques ribereños de Barra de Tecoanapa, Guerrero; durante el periodo de 1990-1995

\begin{tabular}{|c|c|c|c|c|c|c|c|c|c|c|c|c|}
\hline \multirow[t]{2}{*}{ AÑO } & \multicolumn{2}{|c|}{1990} & \multicolumn{2}{|c|}{1991} & \multicolumn{2}{|c|}{1992} & \multicolumn{2}{|c|}{1993} & \multicolumn{2}{|c|}{1994} & \multicolumn{2}{|c|}{1995} \\
\hline & $F$ & $P$ & $F$ & $P$ & $F$ & $P$ & $F$ & $P$ & $F$ & $P$ & $F$ & $P$ \\
\hline Enero & & & 109 & 0 & 105 & 1 & 58 & 2 & 189 & 1 & 103 & 0 \\
\hline Febrero & & & 82 & 0 & 66 & 0 & 67 & 1 & 101 & 2 & 72 & 0 \\
\hline Marzo & & & 114 & 0 & 75 & 0 & 53 & 0 & 90 & 0 & 81 & 0 \\
\hline Abril & & & 88 & 1 & 33 & 0 & 21 & 0 & 86 & 3 & 49 & 0 \\
\hline Mayo & & & 109 & 1 & 59 & 1 & 90 & 4 & 102 & 2 & 93 & 1 \\
\hline Junio & 82 & 1 & 112 & 2 & 96 & 2 & 102 & 6 & 116 & 3 & 111 & 1 \\
\hline Julio & 78 & 3 & 104 & 3 & 160 & 5 & 177 & 4 & 95 & 6 & 157 & 7 \\
\hline Agosto & 102 & 1 & 119 & 1 & 87 & 6 & 179 & 2 & 102 & 2 & 136 & 4 \\
\hline Septiembre & 144 & 0 & 89 & 1 & 152 & 18 & 136 & 4 & 124 & 3 & & \\
\hline Octubre & 92 & 0 & 197 & 2 & 147 & 11 & 131 & 5 & 129 & 3 & & \\
\hline Noviembre & 136 & 0 & 131 & 1 & 73 & 8 & 103 & 4 & 97 & 5 & & \\
\hline Diciembre & 108 & 0 & 123 & 0 & 122 & 6 & 138 & 1 & 117 & 2 & & \\
\hline Total & 742 & 5 & 1377 & 12 & 1175 & 58 & 1255 & 33 & 1348 & 32 & 802 & 13 \\
\hline Prom./año & 106 & 0.7 & 114.8 & 1 & 97.9 & 4.8 & 104.6 & 2.8 & 112.3 & 2.7 & 100.3 & 1.6 \\
\hline$D E$ & 25.6 & 1.1 & 29.8 & 1 & 40.3 & 5.5 & 49.6 & 2 & 27.6 & 1.6 & 34.8 & 2.6 \\
\hline Error Est. & 9.6 & 0.4 & 8.5 & 0.2 & 11.6 & 1.5 & 14.3 & 0.5 & 7.9 & 0.4 & 12.2 & 0.9 \\
\hline
\end{tabular}

Tabla 2. Producción promedio de flores y

R. mangle, en dos bosques de Barra de Tecoanapa Guerrero, México en el periodo de 1990-1995

\begin{tabular}{|c|c|c|}
\hline PRODUCCIÓN & NÚMERO DE FLORES & $\begin{array}{l}\text { NÚMERO DE } \\
\text { PROPÁGULOS }\end{array}$ \\
\hline Mensual/árbol* & 945 intervalo (187-2 234) & intervalo (0-255) \\
\hline Anual/árbol & 11340 & 276 \\
\hline Anual/hectárea & 11571428 & 281632 \\
\hline
\end{tabular}

* árboles >8 cm DAP, altura promedio $10.6 \mathrm{~m}$ 
En marzo de 1993, se cuantificó la menor cantidad de flores por inflorescencia con 3.0 y enero fue el más abundante con 8.0. En noviembre se presentó la tasa de conversión más alta entre flores cerradas y propágulos formados con 3.8:1. Por el contrario, en enero se presentó la más baja con 9.4:1, como se observa en la Tabla 3.

El desarrollo de los propágulos desde su formación hasta su maduración, se llevó a cabo en 226 días (DE 29), hasta que se desprendieron del árbol parental. Los propágulos desarrollados durante mayo y septiembre presentaron las tasas de crecimiento y maduración más rápida en comparación con los de noviembre, enero y marzo. La duración total del proceso, desde que se forman las flores hasta que caen los propágulos, fue de 357 días (DE 21). El periodo más corto observado fue el de las flores formadas en septiembre de 1993, con 329 días; y el más largo el de noviembre, con 379 días. De 2043 flores marcadas, 1032 fueron polinizadas, a partir de ellas se produjeron 596 frutos, de estos emergieron 394 propágulos y maduraron 378 . Los resultados del análisis de Spearman indican las correlaciones significativas, como se observa en la Tabla 4.

Tabla 3. Flores cerradas, polinizadas, frutos, propágulos y duración de cada evento en días

\begin{tabular}{|c|c|c|c|c|c|c|}
\hline MESES & $\begin{array}{c}\text { FLORES } \\
\text { MARCADAS } \\
\text { A }\end{array}$ & $\begin{array}{c}\text { FLORES } \\
\text { POLINIZADAS } \\
\text { B }\end{array}$ & $\begin{array}{c}\text { FRUTOS } \\
\text { FORMADOS } \\
\text { C }\end{array}$ & $\begin{array}{c}\text { PROPÁGULOS } \\
\text { FORMADOS } \\
\text { D }\end{array}$ & $\begin{array}{c}\text { PROPÁGULOS } \\
\text { CAÍDOS } \\
\text { E }\end{array}$ & $\begin{array}{l}\text { PERIODO } \\
\text { TOTAL EN } \\
\text { DÍAS }\end{array}$ \\
\hline Mar & 384 & $204 / 56^{\star}$ & $120 / 79^{*}$ & $61 / 241^{*}$ & $59 / 376^{\star}$ & 376 (DE 24) \\
\hline May & 389 & $223 / 49$ & $121 / 75$ & $82 / 211$ & 79/335 & 335 (DE 33) \\
\hline Jul & 299 & $142 / 61$ & $91 / 82$ & $67 / 215$ & $65 / 358$ & $358(D E 31)$ \\
\hline Sep & 274 & $132 / 54$ & $88 / 69$ & $55 / 206$ & $51 / 329$ & 329 (DE 39) \\
\hline Nov & 289 & $153 / 58$ & $112 / 77$ & $80 / 244$ & $76 / 379$ & 379 (DE 22) \\
\hline Ene & 408 & $178 / 55$ & $64 / 71$ & $49 / 240$ & $48 / 366$ & 366 (DE 29) \\
\hline Total & 2043 & $1032 / 333$ & $596 / 453$ & $394 / 1357$ & $378 / 2143$ & \\
\hline Prom. & 340.5 & $172 / 56$ & $99.3 / 76$ & $65.7 / 226$ & $63 / 357$ & \\
\hline D. Est. & 59.3 & $36.1 / 4$ & $22.4 / 4.9$ & 13.3/17.3 & $12.8 / 21$ & \\
\hline
\end{tabular}

En negritas: número de días en que sucedió el evento

Tabla 4. Correlación de Spearman para flores cerradas, polinizadas, fruto $\mathrm{P}<0.05$

\begin{tabular}{|c|c|c|c|c|}
\hline PARES DE VARIABLES & $\mathrm{N}$ & SPEARMAN $R$ & $\mathrm{~T}(\mathrm{~N}-2)$ & $\begin{array}{l}\text { PROBA- } \\
\text { BILIDAD }\end{array}$ \\
\hline Flores marcadas vs flores polinizadas & 7 & 0.8571 & 3.7210 & 0.01369 \\
\hline Flores polinizadas vs frutos formados & 7 & 0.7857 & 2.8401 & 0.03623 \\
\hline Frutos formados vs propágulos formados & 7 & 0.8928 & 4.4332 & 0.00680 \\
\hline Frutos formados vs propágulos caídos & 7 & 0.8928 & 4.4332 & 0.00680 \\
\hline
\end{tabular}


De las flores marcadas, la mayor pérdida se registró estando las flores cerradas; $45 \%$ de ellas fueron desprendidas por el viento, mientras que del total de flores polinizadas se perdió un $41.1 \%$; los frutos se ubicaron en tercer sitio $(38.7 \%)$. Finalmente, del total de propágulos formados, sólo se perdió el $3.9 \%$ durante el crecimiento y maduración. La época seca (enero-marzo) fue la más crítica para los tres primeros arrollo, mientras que entre junio-julio las pérdidas fueron mínimas.

\section{Establecimiento de los propágulos y supervivencia de plantas}

Los propágulos se sembraron con un peso y longitud promedio de $29.9 \mathrm{~g}$ (DE 12.7) y $25.6 \mathrm{~cm}$ (DE 7.2). En todos los lotes, el tiempo de aparición del primer par de hojas en las plantas fue de 17 días (DE 5), desde que se sembraron los propágulos hasta la apertura de los primordios foliares. Se observaron diferencias en la supervivencia de las plantas bajo las dos condiciones de iluminación; sin embargo, estadísticamente no existen diferencias significativas $(-0.625203 \mathrm{p}=0.53184 ; \mathrm{p}<0.05)$ en la mortalidad, la altura y estructura desarrollada por las plantas, ni en el tiempo de la primera floración.

En la figura 2 se observa que el número de plántulas germinadas en ambos lotes fue muy semejante (193 y 191 plántulas, 96\%). Al final del experimento, las plantas supervivientes a la sombra representaron sólo el 16\% (32) de los propágulos iniciales, mientras que de los sembrados a cielo abierto sobrevivieron $28.5 \%$ (57). Durante el primer año, la mayor mortalidad se registró al sol, ya que el $49.5 \%$ (95) perecieron; en comparación con el 38.5\% (74) de aquellas desarrolladas a la sombra. Esta situación se mantuvo en el segundo año, cuando la mortalidad alcanzó $65 \%$ (123) y $50.5 \%$ (95) en cada lote. A los 20 meses, aparecieron las primeras ramas en las plantas expuestas a la luz directa; sin embargo, a la sombra del bosque hasta los 28 meses aparecieron las ramas. Inicialmente, las plantas bajo el bosque presentaron un desarrollo vigoroso, situación que se invirtió al final del segundo año, desarrollándose mejor las expuestas al sol. A los 23 meses aparecieron las primeras raíces aéreas en las plantas expuestas al sol, a partir de este momento las plantas que murieron fueron las que no desarrollaron estas estructuras.

El proceso de floración sólo se registró en el lote expuesto al sol en agosto de 1995, a los 58 meses (Fig. 2B), mientras que a la sombra florecieron 73 meses después de sembrados (enero de 1997). El espaciamiento final entre las plantas al sol y sombra fue de 148.7 y 191.4 $\mathrm{cm}$. La salinidad intersticial en ambos sitios varió desde 0 durante los meses de lluvias hasta $24 \%$ en marzo y abril.

\section{DISCUSIÓN}

En el continente americano existen pocas investigaciones sobre la fenología reproductiva de las especies de mangles (Gill y Tomlinson, 1971; Rabinowitz, 1978; Jiménez, 1988; Domínguez et al., 1998). Fuera de estas referencias, la información somera y fragmentada sobre este tópico proviene de los estudios sobre caída de hojarasca (Day et al., 1996; Tovilla y González, 1994).

La cantidad de flores y propágulos producida por árbol/ha en Barra de Tecoanapa es $43.2 \%$ superior a la observada en Laguna de Alvarado y Términos, en el Golfo de México, en una latitud ligeramente mayor que la de este sistema, con mayor abundancia de precipitaciones y aportes de agua dulce (Tovilla y González, 1994). Esta producción asegura una tasa de regeneración de plantas durante el año más que sobrada. 
A

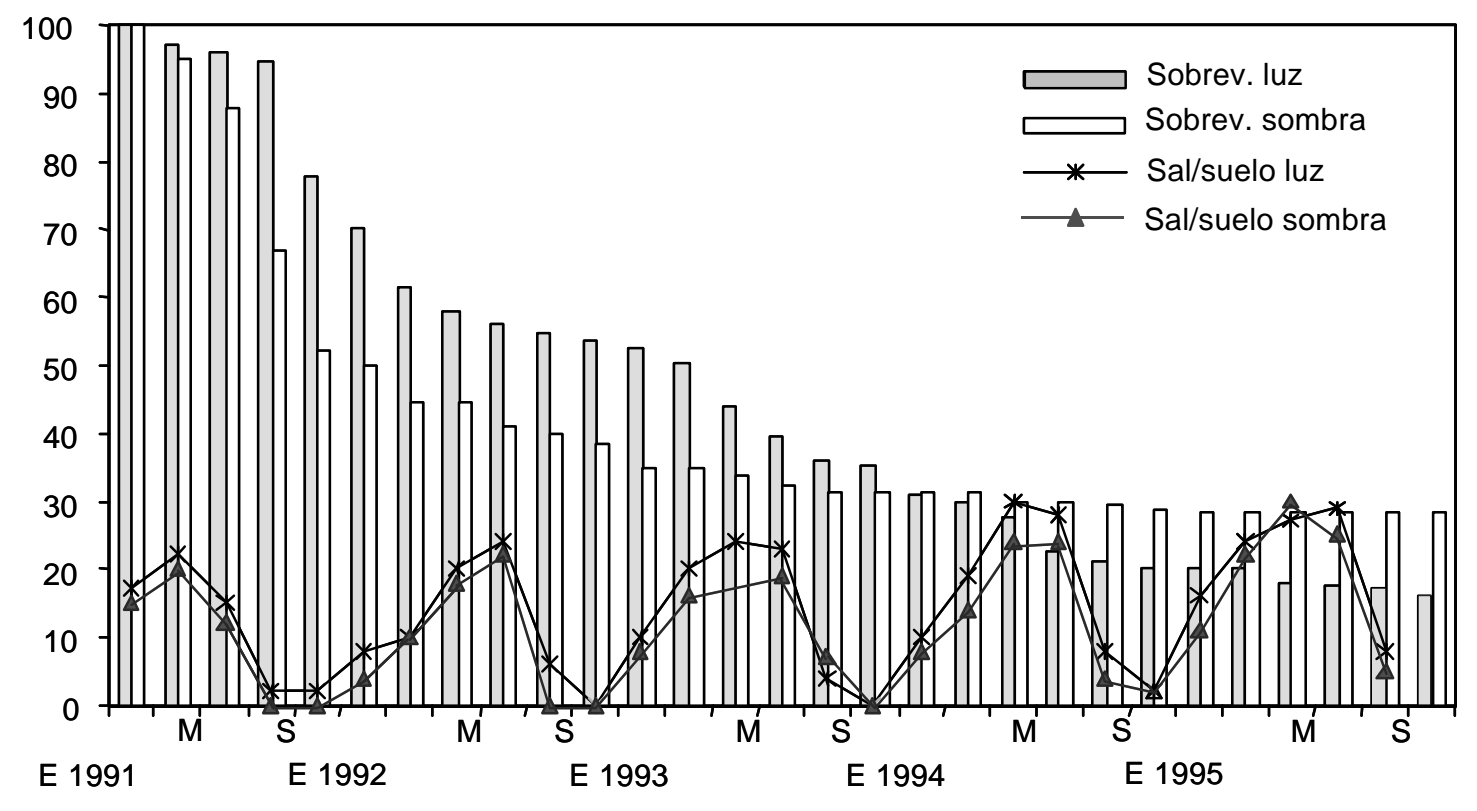

Tiempo (meses)

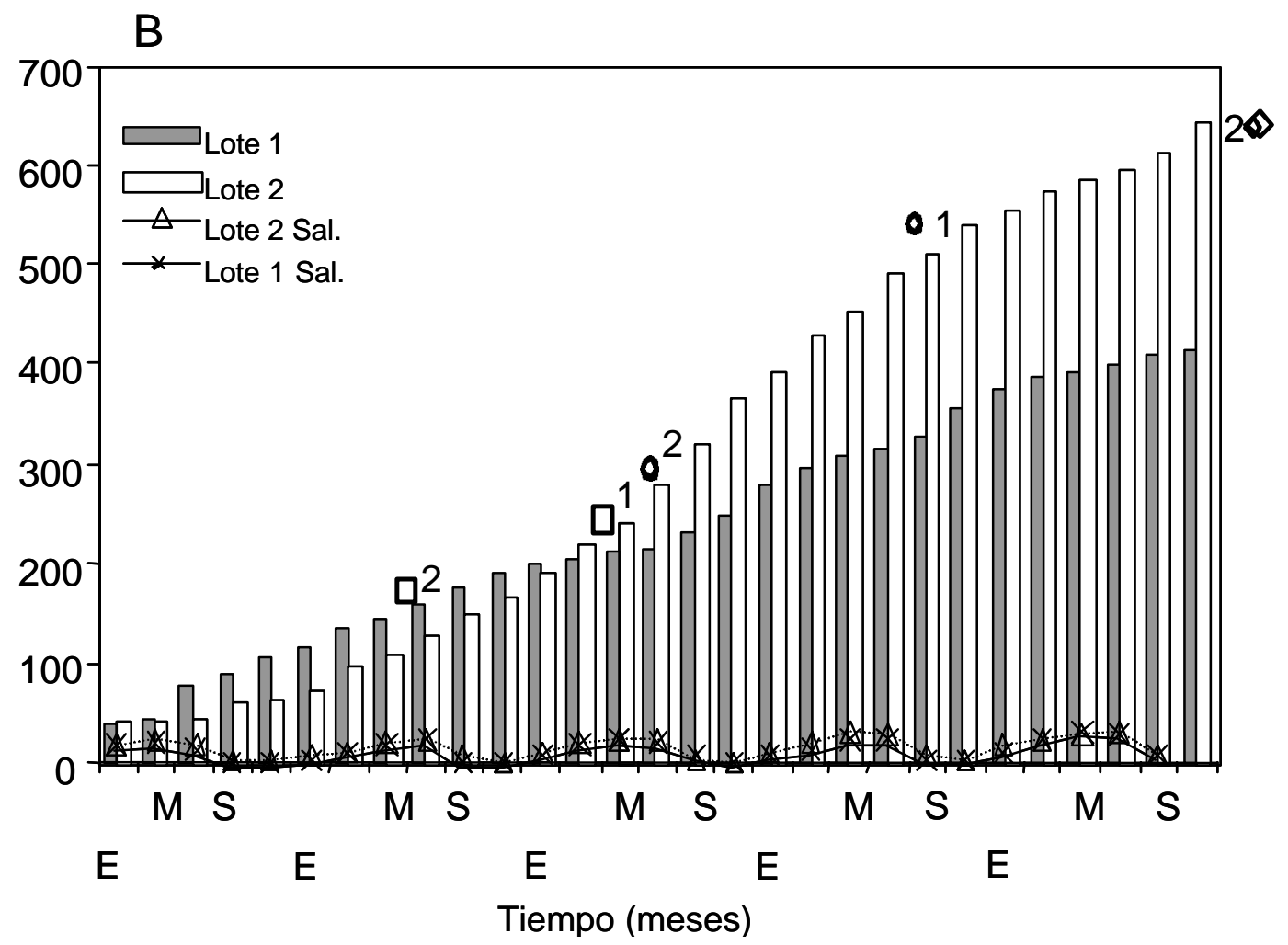

Figura 2. Germinación de hipocotilos: A) sobrevivencia, B) altura alcanzada por dos lotes de plantas de $R$. mangle. Lote1:bajo sombra; lote 2: en un área abierta. 
Esto es más significativo entre la cantidad de propágulos generados entre lluvias y secas donde existe una proporción de 3.1:1. Una explicación es que la producción excesiva de propágulos durante la época de lluvias, no se queda en los bosques, siendo exportada hacia otras áreas del humedal y de la zona marina. Muchos de los propágulos al caer no alcanzan a insertarse en el suelo, debido a que éste se encuentra inundado (más de $30 \mathrm{~cm}$ ), quedando a la deriva, de donde son removidos por las mareas. En la época de lluvias se observó una gran cantidad de propágulos flotando hacia la desembocadura del río, de donde fueron redistribuidos por las corrientes a lo largo de las playas.

La mayoría de los propágulos producidos durante las lluvias son más grandes y pesados $(30.6 \mathrm{~cm}$ y $34.5 \mathrm{~g}$ ), por lo que el embrión dispone de mayor reserva nutritiva, lo que le permite flotar durante más tiempo, con la posibilidad de colonizar nuevas áreas distantes de donde se originaron. Así, los propágulos pueden mantenerse flotando o establecerse sobre cualquier sustrato durante un tiempo razonable sin llegar a dañarse. Por el contrario, los propágulos producidos durante la época de secas, aun cuando son más pequeños $(23.4 \mathrm{~cm}$ y $28.01 \mathrm{~g}$ ), pueden fácilmente insertarse en el fango y germinar cuando caen, debido a que la altura de la columna de agua en el bosque es menor de $5 \mathrm{~cm}$. Este proceso fue observado al interior de los bosques en noviembre y diciembre de todos los años estudiados, donde gran cantidad de propágulos se establecen formando la cohorte de plántulas más importante del año.

Los propágulos en Barra de Tecoanapa son, en promedio, más grandes y pesados que los registrados en Laguna de Términos en Campeche y Laguna de Alvarado en Veracruz, México, así como en Florida, Panamá y Belice. Esta situación es por demás notable debido a que esta localidad presenta menores precipitaciones (Gill y Tomlinson, 1971; Rabinowitz, 1978; Smith y Snedaker, 1995; McKee, 1995b; Tovilla y González, 1994; Tovilla, 1998) como se observa en la Tabla 5.

R. mangle despliega una serie de estrategias para asegurar su permanencia en los bosques durante la época seca y posibilita la colonización de nuevas áreas con los propágulos producidos en lluvias. La abundancia de propágulos durante todo el año puede ser utilizada para facilitar la recuperación de áreas alteradas cercanas a los canales de marea.

Tabla 5. Longitud y peso de propágulos de R. mangle en Panamá, Florida, Belice y México

\begin{tabular}{lcccl}
\hline LUGAR & $\begin{array}{c}\text { LONGITUD } \\
(\mathrm{cm})\end{array}$ & $\begin{array}{c}\text { PESO } \\
(\mathrm{g})\end{array}$ & $\begin{array}{c}\text { TAMAÑO } \\
\text { MUESTRA }\end{array}$ & REFERENCIA \\
\hline Panamá & $22.1 \pm 1$ & $14.0 \pm 1.1$ & 50 & Rabinowitz (1978) \\
\hline Florida & $16.5 \pm 1$ & $12.3 \pm 1.2$ & 50 & $\begin{array}{l}\text { Smith y Snedaker } \\
(1995)\end{array}$ \\
\hline Belice & $23.5 \pm 0.6$ & $20.2 \pm 0.3$ & 97 & McKee (1995b) \\
\hline $\begin{array}{l}\text { Laguna de Términos, } \\
\text { México }\end{array}$ & $20.3 \pm 3.8$ & $19.5 \pm 5.1$ & 250 & $\begin{array}{l}\text { Tovilla y González } \\
(1994)\end{array}$ \\
\hline $\begin{array}{l}\text { Laguna de Alvarado, } \\
\text { México }\end{array}$ & $19.8 \pm 5.4$ & $18.4 \pm 4.1$ & 150 & $\begin{array}{l}\text { Tovilla y González } \\
\text { (1994) }\end{array}$ \\
\hline $\begin{array}{l}\text { Barra de Tecoanapa, } \\
\text { Guerrero }\end{array}$ & $25.6 \pm 7.8$ & $29.9 \pm 8.9$ & 2631 & Tovilla (1998) \\
\hline
\end{tabular}


La floración y producción de propágulos está asociada a la estacionalidad y a la frecuencia de vientos fuertes que se dejan sentir entre enero y abril en el área, factores que desprenden una gran cantidad de flores cerradas, polinizadas y frutos de las ramas en estos meses. Es posible que la insolación y temperaturas mayores a $28 \stackrel{\circ}{\circ}$ favorezcan ampliamente la floración, como lo han demostrado algunos estudios realizados en Florida y Costa Rica (Gill y Tomlinson, 1971; Jiménez, 1988). De acuerdo con Jiménez (1988), la eficiencia en el proceso de floración y producción de propágulos está relacionada también con otros factores ambientales como la presencia de lluvias, vientos, insolación, sequía y temperatura, como las que existen en el área de estudio. En noviembre, al inicio de la época seca, con una salinidad de $10 \%$-12\%; $28{ }^{\circ} \mathrm{C} ; 9.5$ horas de insolación/día; $69 \%$ de humedad y ausencia de vientos (CNA, 1995), se registró la tasa de conversión más alta con 3.8 flores por cada propágulo maduro. Por el contrario, en enero cuando la salinidad era de 24\%; con temperatura de $31^{\circ} \mathrm{C}, 10.5$ horas/día de insolación, $55 \%$ de humedad y vientos moderados ( $4 \mathrm{~m} / \mathrm{s}$ - $5 \mathrm{~m} / \mathrm{s}$ ), la tasa de conversión fue baja 9.4:1.

Gill y Tomlinson (1971) con salinidad de $18 \%$ a $20 \%$; temperatura de $22{ }^{\circ} \mathrm{C}$; 8.5 horas de sol; $95 \%$ de humedad y vientos débiles, sólo registraron un $6.6 \%$ de flores polinizadas en $R$. mangle y de estas flores, sólo el $23.8 \%$ se convirtieron en propágulos maduros, una tasa tan baja como 269.4 flores por cada propágulo maduro; en Barra de Tecoanapa esta conversión fue de 49 flores/propágulo maduro. En Florida, Gill y Tomlinson (1971) registraron la máxima floración en esta especie en mayo-julio (28 flores $/ \mathrm{m}^{2}$ ). Esta producción representa menos de la tercera parte de la floración observada en el mismo periodo de tiempo en este trabajo.
De acuerdo con McKee (1995b), los manglares poseen una limitada capacidad para la reproducción vegetativa, por lo que dependen exclusivamente del proceso de arraigamiento de los propágulos para sobrevivir. En este bosque, el establecimiento de los propágulos se realizó en un tiempo breve (17 días $D E 5)$; mientras que Rabinowitz (1978) observó que, en Panamá, este fenómeno sucedía en nueve días en agua dulce y en 30 en agua salada. McKee (1995c) indica que el proceso de arraigo temprano de los propágulos está más relacionado con la disponibilidad de nutrientes en el medio que con la luz. Aquí se registró una elevada germinación (96\%); este porcentaje pudo ser mayor (99.5\%) restando el consumo de los cangrejos Goniopsis pulchra y Cardisoma crassum, los cuales son abundantes entre enero y marzo.

La mortalidad al sol, está relacionada con la frecuencia de lavado y humectación que ejercen las mareas a lo largo del año. Este sitio presentó un lavado promedio por las mareas durante 9 meses (540 veces/año). Ellison y Farnsworth (1993), en Belice, observaron que $R$. mangle presentaba mayor sobrevivencia relacio-nada con una mayor influencia de las mareas presentes en cada sitio, indistin-tamente si las plántulas estuvieran bajo el dosel del bosque o al sol. En Tecoanapa, las plantas desarrolladas al sol, desde muy temprana edad presentaron una fuerte competencia por espacio y después de 24 meses, las plantas sobrevivientes eran más altas y vigorosas que las de sombra. Al final, las únicas plantas supervivientes fueron aquellas que ganaron altura rápidamente. De manera semejante, Farnsworth $y$ Ellison (1993) observaron que en Belice, $R$. mangle presenta una elevada flexibilidad a tolerar una gran iluminación cuando las plantas son jóvenes; posteriormente, cuando las plantas se vuelven adultas sus requerimientos de luz van disminuyendo. 
La aparición de las raíces aéreas es el penúltimo evento que aumenta su esperanza de vida, ya que además de aumentar rápidamente el grosor de los tallos, aumenta el área basal en forma notable, lo que permite captar mayor cantidad de luz y nutrientes del suelo y limitar el desarrollo de otras plantas cercanas.

La floración en Tecoanapa fue más rápida que en plantaciones de mangles en el sudeste asiático, donde se ha registrado la floración después de 75 meses (FAO, 1994). Contrariamente a esto, Ellison y Farnsworth (1997) obtuvieron la floración en $R$. mangle a los 18 y 26 meses bajo condiciones de invernadero y fertilizando las plantas cada dos semanas. La aparición de las raíces aéreas también disminuyó la mortalidad de las plantas a la sombra, aunque el desarrollo, estructural y en altura, fue lento. A la sombra, las únicas plantas que alcanzaron la floración estaban ubicadas en donde se abrió un claro en el bosque al ser derribado un árbol. Una situación semejante fue observada por Rabinowitz (1978) y McKee (1995b) con plantas desarrolladas a la sombra en Panamá y Belice, donde más del $60 \%$ de plántulas de $R$. mangle murieron después de 30 meses.

\section{CONCLUSIÓN}

La fenología reproductiva de $R$. mangle en el área de estudio está determinada por la presencia de lluvias. Los años secos disminuyen fuertemente la cantidad de flores y propágulos, el tamaño y peso de ellos; los años lluviosos producen cambios sustanciales en la reserva genética de los bosques. Con una proporción de flores/propágulos de 49:1, la producción de propágulos por árbol/ año en el campo es baja, lo cual se acentúa aún más durante los años secos. Un ciclo de floración se completa en 357 días, de los cuales, los primeros 135 representan la etapa más crítica de la floración. Durante este lapso se pierde hasta un $70.8 \%$ de las flores iniciales, mientras que la última parte del desarrollo de los propágulos es muy exitoso.

La viabilidad de los propágulos es muy alta (más de 96\%) debido a la abundante reserva de nutrientes del propágulo, de la cual depende durante el primer año de vida. En los primeros 18 meses, la mayor mortalidad se registró al sol, por la excesiva desecación de los propágulos. La aparición de las primeras raíces aéreas limita fuertemente la mortalidad debido a que permite a las plantas explotar los nutrientes de un área más grande en el suelo y fortalece la estructura arbórea de las copas, para competir por espacio con el resto de las plantas cercanas. $R$. mangle requiere hasta 4.8 años para alcanzar la primera floración expuesta al sol, mientras que a la sombra pocos árboles la alcanzaron a

\section{AGRADECIMIENTOS}

Los autores agradecen a los biólogos Eladio González Angelito y Enoch González Angelito así como al Técnico Hipólito Bibiano y a la Señora Minerva Pérez por la asistencia en el trabajo de campo, sin la cual esta investigación no hubiera sido posible.

\section{REFERENCIAS}

Cintrón G.M. y Y. Schaeffer N. 1981. Roteiro para estudio dos recursos de marismas e manguezais. Boletín Instituto Oceanográfico de Sao Paulo. 10:1-13.

Clarke P.J., 1995. The population dynamics of the mangrove Avicennia marina L; demographic synthesis and predictive modelling. Hydrobiologia 295:83-88. 
Clarke, P.J. y W.G. Allaway. 1993. The regeneration niche of the grey mangrove (Avicennia marina L.), effects of salinity, light and sediments factors on establishment, growth and survival in the field. Oecologia 93: 548-556.

Comisión Nacional del Agua (CNA). 1995. Algunas modificaciones ambientales en las costas del Pacífico de México, Documento Interno No. 11-A, 38pp. México.

Chapman, V.J. 1974. Mangrove biogeography. In: Walsh, G.; S. Snedaker, y $H$. Teas, eds. Proc. Internatl. Symp, Biol. Mangt. Mangroves. Honolulu, Hawaii. pp:3-22.

Christensen, B. 1978. Biomass and primary production of Rhizophora apiculata BI. in a mangrove southern Thailand. Aquatic Botany 4:43-52.

Day Jr. J.W.; C. Coronado M.; F.R. Vera H.; R. Twilley; V. Rivera M.; H. Alvarez G.; R. Day y W. Conner. 1996. A 7 year record of above-ground net primary production in a southeastern Mexican mangrove forest. Aquatic Botany 55:39-60.

Domíguez A., C.; L.E. Eguiarte; J. Núñez F. y R. Dirzo. 1998. Flower morphometry of Rhizophora mangle (Rhizophoraceae): geographical variation in Mexican populations. American Journal of Botany 85(5):637-643.

Ellison A., M. y E.J. Farnsworth. 1993. Seedling survivorship, growth and response to disturbance in Belizean mangroves. American Journal of Botany 80:1137-1145.

Ellison A., M. y E.J. Farnsworth. 1997. Simulated sea level change alters anatomy, physiology, growth, and reproduction of red mangrove
(Rhizophora mangle L.). Oecologia 112:435-446.

FAO (Organización de las Naciones Unidas para la Agricultura y la Alimentación) 1994. Directrices para la organización de los manglares: Estudio FAO Montes 117. Roma Italia.

Farnsworth E., J. y A.M. Ellison. 1993. Sunshade adaptability of the red mangrove, Rhizophora mangle (Rhizophoraceae): Changes through ontogeny at several levels of biological organization. American Journal of Botany 83:1131-1143.

Gill, A.M. y P.B. Tomlinson. 1971. Studies on the growth of red mangrove (Rhizophora mangle L.) 3. Phenology of the shoot. Biotropica 3(2):109-124.

Jiménez, J., A. 1988. Floral and fruiting phenology of trees in a mangrove forest on the dry Pacific coast of Costa Rica. Brenesia 29:33-50.

Lugo, A. y S.C. Snedaker. 1975. Properties of mangrove forest in southern Florida. p:170-211. In: G. Walsh, S. Snedaker $y \mathrm{H}$. Teas (eds.) Proceedings of the international symposium on the biology and management of mangroves. Univ. of Florida Gainesville.

McKee K., L. 1995a. Seedling recruitment patterns in a Belizean mangrove forest: effects of establishment ability and physico-chemical factors. Oecologia 101:448-460.

McKee K., L. 1995b. Mangrove species distribution and propagules predation in Belize: an exception to the dominance-predation hypothesis. Biotropica 27 (3):334-345.

McKee K., L. 1995c. Interspecific variation in growth, biomass partitioning, and defensive characteristics of neotropical mangrove seedlings: response to light 
and nutrient availability. American Journal of Botany 82:299-307.

Mepham R., H. 1983. Mangrove floras of the southern continents. Part I The geographical origin of the Indo Pacific mangrove genera and the development and present status of the Australian mangroves. SudAfrican Journal of Botany 2:1-8.

Rabinowitz, D. 1978. Dispersal properties of mangrove propagules. Biotropica 10:47-57.

Secretaría de Agricultura y Recursos Hidráulicos (SARH). 1987. Volúmenes de agua transportados por los Ríos Nexpa, Quetzalapa y Verde durante los últimos 10 años. Publicación Especial 4. 52 p.

Smith M., S. y S.C. Snedaker. 1995. Salinity responses in two populations of viviparous Rhizophora mangle L. seedling. Biotropica 27 (4):435-440.

Tovilla H., C. 1994. Manglares. In: E.G. De la Lanza y C.M. Cáceres. Lagunas Costeras y el Litoral Mexicano. Universidad Autónoma de Baja California Sur. p:370-423.

Tovilla H., C. y E.A. González. 1994. Producción de hojarasca del manglar en tres sistemas lagunares del Golfo de México y el Pacífico. In: Grandes Temas de la Hidrobiología: Los Sistemas Litorales. UAM Iztapalapa, UNAM 2:87-103.

Tovilla H., C. 1998. Ecología de los bosques de manglar y algunos aspectos socioeconómicos de la zona costera de Barra de Tecoanapa Guerrero, México. Tesis Doctoral, Facultad de Ciencias, UNAM. México. 368 p.»

1 Laboratorio de Ecología de Manglares. El Colegio de la Frontera Sur (Ecosur Tapachula). Carretera Antiguo Aeropuerto km 2.5. Tapachula 30700 Chiapas. c.e.: ctovilla@tap-ecosur.edu.mx.

Manuscrito recibido el 17 de agosto de 2001.

Aceptado el 29 de mayo de 2002.

Este documento se debe citar como:

Tovilla H., C. y D.E. Orihuela B. 2002. Floración, establecimiento de propágulos y supervivencia de Rizophora mangle L. en el manglar de Barra de Tecoanapa, Guerrero, México. Madera y Bosques -102 . 